\title{
ON A THEOREM OF SABIDUSSI
}

\section{CHONG-YUN CHAO}

By a graph $X$ we mean a finite set $V(X)$ called the vertices of $X$, together with a set $E(X)$, called the edges of $X$, consisting of unordered pairs of distinct elements of $V(X)$. We shall indicate unordered pairs by brackets. We denote $G(X)$ as the group of automorphisms of $X$. Each element $\rho$ of $G(X)$ is considered as a permutation of $V(X)$ onto itself, such that $[\rho x, \rho y] \in E(X)$ if and only if $[x, y]$ $\in E(X) . G(X)$ is said to be strongly fixed-point-free if $G(X) \neq\{1\}$, and $\phi x \neq x$ for every $x \in X$ and every $\phi \in G(X)-\{1\}$, where 1 is the identity of $G(X) . G(X)$ is said to be regular if it is strongly fixedpoint-free and transitive on $V(X)$.

Let $G$ be a finite group and $H$ a subset of $G$ which does not contain the identity of $G$. By the color-group $X_{G, H}$ of $G$ with respect to $H$, we mean the graph given by $V\left(X_{G, H}\right)=G$ and $E\left(X_{G, H}\right)=\{[g, g h]$; $g \in G, h \in H\}$. It is known that $X_{G, H}$ is connected if and only if $H$ contains a set of generators of $G$.

Recently, Sabidussi showed [2, p. 802] the following theorem:

Let $G(X)$ act regularly on a graph $X$, then $X$ is isomorphic to either the graph $Y_{0}$ with $V\left(Y_{0}\right)=\left\{x_{1}, x_{2}\right\}, E\left(Y_{0}\right)=\square$, or $X$ is connected and isomorphic to a color-group of $G(X)$ with respect to some set $H$ of generators of $G(X)$.

The purpose of this note is to prove the following theorem by using Sabidussi's result.

THEOREM. There exists no graph with $n$ vertices whose group of automorphisms is transitive and abelian where $n>2$.

Proof. We know that every transitive and abelian permutation group is regular (see [3, p. 12]). By Sabidussi's theorem we only need to consider the connected graphs.

Suppose there existed a connected graph $X$ with $n$ vertices where $n>2$ such that $G(X)$ is regular and abelian, then we would have the order and degree of $G(X)$ equal to $n$. Let $G(X)$ be denoted by $\left\{g_{0}=1, g_{1}, \cdots, g_{n-1}\right\}$. By Sabidussi's theorem, there exists a set $H=\left\{g_{k_{1}}, g_{k_{2}}, \cdots, g_{k_{t}}\right\}$ consisting of generators of $G(X)$, in $G(X)$ where $1 \leqq t \leqq n-1$, and $1 \leqq k_{i} \leqq n-1$ for $i=1,2, \cdots, t$ such that $X \cong X_{G(X), H}$. That is $V\left(X_{G(X), H}\right)=\left\{g_{0}, g_{1}, \cdots, g_{n-1}\right\}$ and $E\left(X_{G(X), H}\right)=\left\{\left[g_{i}, g_{i} g_{k_{j}}\right]\right.$;

Received by the editors September 13, 1962 and, in revised form, December 29, 
$i=0,1, \cdots, n-1$, and $j=1,2, \cdots, t\}$. That means the edges incident with each $g_{i}$ are $\left[g_{i}, g_{i} g_{k_{j}}\right]=\left[g_{i}, g_{k_{j}} g_{i}\right]$ and $\left[g_{i} g_{k_{j}}^{-1}, g_{i}\right]=\left[g_{k_{j}}^{-1} g_{i}, g_{i}\right]$ for $i=0,1, \cdots, n-1$, and $j=1,2, \cdots, t$ where commutativity is used.

Let $\rho$ be a permutation of $V\left(X_{G(X), H}\right)$ defined by $\rho\left(g_{i}\right)=g_{i}^{-1}$, $i=0,1, \cdots, n-1$. Since $\left[\rho\left(g_{i}\right), \rho\left(g_{i} g_{k_{j}}\right)\right]=\left[g_{i}^{-1}, g_{k_{j}}^{-1} g_{i}^{-1}\right]$ and $\left[\rho\left(g_{i} g_{k_{j}}^{-1}\right), \rho\left(g_{i}\right)\right]=\left[g_{k_{j}} g_{i}^{-1}, g_{i}^{-1}\right]$ belong to $E\left(X_{G(X), H}\right)$ if and only if

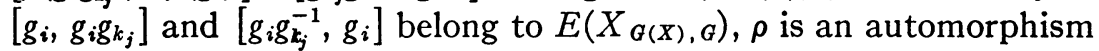
of $X_{G(X), H}$. Since $G(X)$ is strongly fixed-point-free, $\rho=1$. Hence, every nonidentity element in $G(X)$ is of order two, and $G(X) \cong C_{2} \times C_{2}$ $\times \cdots \times C_{2}$, where $C_{2}$ is the cyclic group of order 2 . Since $n>2$, the number of generators in $H$ must be $>1$. Consequently, there is a nonidentity permutation $\sigma$ of $V\left(X_{G(X), H}\right)$ which leaves $g_{0}$ fixed and $\sigma H=H$. Since each element of $G(X)$ can be written as $g_{k_{1}}^{\lambda_{1}} g_{k_{2}}^{\lambda_{2}} \cdots g_{k_{m}}^{\lambda_{m}}$ where $g_{k_{i}}$ are generators of $G(X), m>1$, and $\lambda_{i}=0$ or $1(i=1,2, \cdots, m)$, and since every such product is an element of $G(X)$, it is easy to see that $\sigma$ is an automorphism of $X_{G(X), H}$. Since $\sigma\left(g_{0}\right)=g_{0}$ and $X_{G(X), H}$ $\cong X, G(X)$ is not strongly fixed-point-free. That is a contradiction and the proof is completed.

COROLlaRY 1 (KAGNo [1]). There exists no graph with $n$ vertices whose group of automorphisms is the cyclic group generated by the cycle $(12 \cdots n)$ for $n>2$.

Proof. Since the cyclic group generated by the cycle $\left(\begin{array}{lll}1 & 2 \cdots n\end{array}\right)$ is a transitive and abelian group, apply the theorem to complete the proof.

COROLlaRy 2. There exists no graph with $n$ vertices whose group of automorphisms is regular where $n$ is an odd prime.

Proof. Since $n$ is an odd prime and since the order of $G(X)$ is $n$, $G(X)$ is a cyclic group. Apply Corollary 1 to complete the proof.

I am grateful to Professor Sabidussi for pointing out Kagno's paper, and for putting the theorem in present form.

\section{REFERENCES}

1. I. N. Kagno, Linear graphs of degree $\leqq 6$ and their groups, Amer. J. Math. 68 (1946), 505-520.

2. G. Sabidussi, On a class of fixed-point-free graphs, Proc. Amer. Math. Soc. 9 (1958), 800-804.

3. H. Wieland, Permutation groups, California Inst. of Tech., Pasadena, Calif.

INTERNATIONAL BUSINESS MACHINES 